\title{
The cerebro-cardiovascular response to periodic squat-stand maneuvers in healthy subjects: a time-domain analysis
}

SC Barnes ${ }^{1}, \mathrm{~N}$ Ball1${ }^{1}$, VJ Haunton, 2 TG Robinson ${ }^{1,2}$, RB Panerai1, 2

${ }^{1}$ Department of Cardiovascular Sciences, University of Leicester, Leicester, UK.

${ }^{2}$ National institute for Health Research (NIHR) Leicester Biomedical Research Centre, University of Leicester, Leicester, UK.

Corresponding author:

Victoria J. Haunton

Department of Cardiovascular Sciences

Room 210, Robert Kilpatrick Clinical Sciences Building

University of Leicester

PO Box 65

Leicester LE2 7LX

Email: vjh12@le.ac.uk

Phone: 01162525802

Running title: The physiological effects of squat-stand maneuvers 


\section{Abstract}

Background: Squat-stand maneuvers (SSMs) have been used to improve the coherence of transfer function analysis (TFA) estimates during the assessment of dynamic cerebral autoregulation (dCA). There is a need to understand the influence of peripheral changes resulting from SSMs on cerebral blood flow, which might confound estimates of dCA.

Methods: 29 healthy subjects underwent recordings at rest (5 min standing) and 15 SSMs $(0.05 \mathrm{~Hz})$. Heart rate (3-lead ECG), end-tidal $\mathrm{CO}_{2}$ (capnography), blood pressure (Finometer), cerebral blood velocity (CBV, transcranial Doppler, $\mathrm{MCA}$ ) and the angle of the thigh (tilt sensor) were measured continuously. The response of CBV to SSMs was decomposed into the relative contributions of mean arterial pressure (MAP), resistance area product (RAP) and critical closing pressure (CrCP).

Results: Upon squatting, a rise in MAP $(83.6 \pm 21.1 \%$ contribution $)$ is followed by increased CBV. A dCA response can be detected, determined by adjustments in RAP and CrCP (left hemisphere) with peak contributions of $24.8 \pm 12.7 \%$ and $27.4 \pm 22.8 \%$, respectively, at different times during SSMs. No interhemispheric differences were detected. During standing, the contributions of MAP, RAP and CrCP change considerably.

Conclusions: The changes of CBV subcomponents during repeated SSMs indicate a complex response of $\mathrm{CBV}$ to SSMs that can only be partially explained by myogenic mechanisms. More work is needed to clarify the potential contribution of other co-factors, such as breath-to-breath changes in $\mathrm{pCO}_{2}, \mathrm{HR}$, stroke volume and the neurogenic component of dynamic CA. 
New and Noteworthy

We describe the different contributions to the cerebral blood flow response following squatstand maneuvers, and furthermore we demonstrate the complex interaction of peripheral and cerebral parameters for the first time. Moreover, we show that the CBV response to squatting is likely to include a significant metabolic component.

Keywords

cerebral autoregulation, cerebral blood flow, cerebral haemodynamics, critical closing pressure, transcranial Doppler. 


\section{Introduction}

Dynamic cerebral autoregulation (dCA) was first proposed by Aaslid, who used thigh-cuff deflation to induce step changes in mean arterial pressure (MAP) (2). Subsequently, it was demonstrated that dCA could also be assessed using the spontaneous fluctuations in blood pressure (BP) that occur naturally at rest, using transfer function analysis (TFA) $(26,28,50)$. However, concerns about the low levels of coherence reported in these studies, and the limited reproducibility of estimated parameters led to a search for methods that could induce changes in MAP larger than those occurring spontaneously (50). Proposed approaches included periodic breathing (13), oscillatory lower-body negative pressure (3), the Valsalva manoeuvre (42) and the cold-pressor test (23), with each technique having advantages and disadvantages in terms of feasibility, tolerability, and efficacy.

Recently, repeated squat-stand maneuvers (SSMs) have also been proposed as a suitable approach to assess dCA due to large changes in MAP induced by the manoeuvre $(10,37)$. SSMs have been used to induce BP oscillations at a variety of frequencies $(4,10,35-38,48)$, and have been verified as a safe, effective and reliable method of altering BP for the assessment of dCA that can improve the reproducibility of autoregulatory indices (37). However, whilst much has been written about the various physiological mechanisms that mediate the physiological response to the $\operatorname{SSM}(20,24,32,34)$, the influence of concurrent peripheral and cerebrovascular physiological changes induced by the SSM on estimates of dCA have not been described. In particular, the extent to which the dynamic relationship between MAP and cerebral blood flow (CBF), usually estimated as CBF velocity (CBV) with transcranial Doppler (TCD) (27), truly reflects myogenic regulatory control of CBF has not been investigated. Analysis of SSM recordings is usually performed in the frequency domain, based on TFA (26), which does not allow for the identification of the peripheral and cerebrovascular influences on CBF. Examples of possible sources of interference would be the baroreflex, autonomic nervous system activity, the muscle mechanoreflex, and changes in breathing frequency and corresponding $\mathrm{pCO}_{2}$ levels. 
To our knowledge, no study has considered the continuous response of both the cerebrovasculature and the cardiovascular system to SSMs, and no study has considered the response of CBV subcomponents to SSMs. The aims of this study were to quantify the influence of the SSM on cerebrovascular parameters, and to determine the extent to which the CBF response to the SSM can be considered as purely myogenic. Using a time domain analysis of the subcomponents that could influence CBV during SSMs, we tested the hypothesis that the MAP-CBV relationship does not represent a purely myogenic response of the cerebral circulation.

\section{Methods and materials}

This study was based on a sub-set of data, collected between September 2016 and January 2017 that were part of a wider study. For the purposes of the present study, we have focused on recordings performed in healthy subjects standing and performing SSM at a frequency of $0.05 \mathrm{~Hz}$.

\section{Ethical approval}

Ethical approval was obtained from the University of Leicester (Ref: vjh-8442) and all participants provided written consent. Our inclusion criteria were as follows; male or female aged 18 years or over who were able and willing to comply with all study requirements and had given their informed consent. Females who were pregnant or lactating were excluded.

\section{Instrumentation}

CBV was recorded bilaterally with TCD (Viasys Companion III) in the middle cerebral artery (MCA), using $2 \mathrm{MHz}$ probes held in place using a head-frame. Heart rate $(\mathrm{HR})$ was recorded using 3-lead ECG, end-tidal $\mathrm{CO}_{2}$ via nasal capnography, (Salter Labs, ref. 4000; Capnocheck Plus) and continuous beat-to-beat blood pressure (BP) using arterial volume-clamping of the digital artery on the left hand (Finometer, Finapres Medical Systems; Amsterdam, the Netherlands). The hand was held in position with a sling to minimise movement throughout the recordings, and the servo-reset mechanism was disabled throughout the recordings to allow for a continuous BP trace, but enabled between recordings. The brachial BP (UA767 
BP monitor) of the dominant arm was measured prior to each recording for calibration of the Finometer device. A tilt-sensor (QG-KI-090AI-K, DIS Sensors, Oostergacht, The Netherlands) was attached to the subject's right thigh with a Velcro strap $20 \mathrm{~cm}$ above the superior border of the patella to measure the angle of the squatting motion.

Data from the above parameters were simultaneously recorded onto the Physiological Data Acquisition System (PHYSIDAS) designed by the Leicester Medical Physics group for subsequent offline analysis.

\section{Experimental procedures}

The protocol took place in the Cerebral Haemodynamics in Ageing and Stroke Medicine (CHIASM) laboratory at the Leicester Royal Infirmary, which is of controlled temperature, welllit, and free from distraction. Participants were asked to avoid strenuous exercise, caffeine, smoking, large meals and alcohol in the four hours prior to their visit.

Once the participant was prepared and satisfactory signals were obtained from all instruments, a 5 min recording was taken with the subject standing quietly with their eyes open. This was followed by 15 repeated SSMs at a frequency of $0.05 \mathrm{~Hz}$, preceded and followed by 90 seconds standing. A computer program provided the participant with visual cues to guide the timing of the maneuvers.

Each recording was made with minimal background distraction to minimise the effects of auditory stimulation on CBV. A period of instruction and practice preceded the second recording, during which the SSM was demonstrated. When performing SSMs, subjects were instructed to squat down as low as they felt able. They were informed that they would need to perform 15 squats, and to take this into account when choosing their depth. Throughout each recording, subjects were asked to breathe through their nose and to avoid a Valsalva-like manoeuvre during the SSM.

\section{Data processing}

The readings from the Finometer were calibrated to the brachial BP recordings. Data were visually inspected; non-physiological spikes in CBV were removed through linear interpolation. 
Files that contained any segments of significantly poor TCD signals were excluded from further analysis. Narrow spikes ( $<100 \mathrm{~ms})$ and artefacts were removed by linear interpolation. Subsequently, all signals were filtered in the forward and reverse direction using an eighthorder Butterworth low-pass filter with a cut-off frequency of $20 \mathrm{~Hz}$. The beginning and end of each cardiac cycle were detected in the BP signal, and mean values of BP, CBV and HR were obtained for each heartbeat. The critical closing pressure (CrCP) and resistance-area product (RAP) were estimated with the first harmonic method (25). Beat-to-beat parameters were interpolated with a third-order polynomial and resampled at $5 \mathrm{~Hz}$ to generate signals with a uniform time base.

The moment at which each subject initiated their 15 SSMs was marked manually during data editing, using a custom-designed computer program. Intra-, followed by inter-subject, averaging generated the population coherent average SSM for the cohort, as well as the intersubject standard deviation (SD) for each instant of time, synchronised by the beginning of the squat manoeuvre. To quantify the CBV response to SSM, we employed sub-component analysis as described below.

\section{Subcomponent analysis}

Similar to the lung, coronary and other circulations, the cerebral circulation shows the existence of a $\mathrm{CrCP}$, as the perfusion pressure where blood flow stops $(8,12,14,25)$. To account for the existence of a $\mathrm{CrCP}>0$, instantaneous pressure-flow (or velocity) relationships can be expressed as:

$$
V_{0}=\frac{M A P_{0}-C r C P_{0}}{R A P_{0}}
$$

where RAP describes the resistance-area product (15) and the subscript ' 0 ' indicates the operating point at baseline.

According to equation [1], changes in CBV can be explained by corresponding changes in its subcomponents, that is $\mathrm{BP}, \mathrm{CrCP}$ or RAP. Assuming that a change in $\mathrm{BP}$ will lead to 
accompanying changes in $\mathrm{CrCP}, \mathrm{RAP}$, and hence CBV, it is possible to demonstrate that the percent change in CBV can be expressed as the corresponding percent changes in its subcomponents (30), that is:

$V_{0}+\Delta v=\frac{\left(M A P_{0}+\Delta p\right)-\left(C r C P_{0}+\Delta c\right)}{\left(R A P_{0}+\Delta r\right)}$

where $\Delta v, \Delta p, \Delta c$ and $\Delta r$ represent small changes in CBV, MAP, CrCP and RAP, respectively.

Expanding and simplifying [2] gives:

$\Delta v=\frac{\Delta p-\Delta c-V_{0} \Delta r}{R A P_{0}+\Delta r}$

Dividing through by $\mathrm{V}_{0}$, to calculate the relative magnitude of $\Delta \mathrm{v}$, yields:

$\frac{\Delta v}{V_{0}}=\frac{\Delta p-\Delta c-V_{0} \Delta r}{V_{0}\left(R A P_{0}+\Delta r\right)}$

Thus the contribution of each subcomponent to $\Delta \mathrm{v}$ is defined as:

$V_{M A P}=\frac{\Delta p}{V_{0}\left(R A P_{0}+\Delta r\right)}$

$V_{C r C P}=-\frac{\Delta c}{V_{0}\left(R A P_{0}+\Delta r\right)}$

$V_{R A P}=-\frac{\Delta r}{\left(R A P_{0}+\Delta r\right)}$

Where

$\Delta v=V_{M A P}+V_{C r C P}+V_{R A P}$

Values of $\mathrm{V}_{0}$ and $\mathrm{RAP}_{0}$ used for normalisation (eq. 1) were extracted at the beginning of each squat manoeuvre, to take into account the dynamic nature of the baseline period (Fig. 1). In most applications, $\Delta r<<R_{0}$, thus making $V_{M A P}$ and $V_{C r C P}$ independent of $\Delta r$.

For the interpretation of the $\mathrm{V}_{\mathrm{CrCP}}$ and $\mathrm{V}_{\mathrm{RAP}}$ terms, it is important to note the negative sign in eqs. [6] \& [7] leads to a positive contribution to $\triangle \mathrm{V}$ when both RAP and CrCP decrease. Conversely, when these parameters increase, they will induce reductions in CBV. Furthermore, previous work suggests that the two subcomponents represent different parts of the $\mathrm{dCA}$ response. $\mathrm{V}_{\mathrm{CrCP}}$ is thought to be representative of the metabolic component of dCA, 
and $\mathrm{V}_{\mathrm{RAP}}$ may reflect the myogenic component (29). These presumed associations will be further addressed in the Discussion.

The utility of subcomponent analysis has been previously demonstrated for physiological studies, as well as for the interpretation of cerebrovascular alterations in patients with clinical conditions including stroke and pre-eclampsia $(22,29,30,33,44)$.

\section{Statistical analysis}

Data are given as means \pm SD. Paired Student's t-tests were used to compare data from the left and right brain hemispheres, and between the two recordings. Significance was set at $\mathrm{p}<0.05$.

\section{Results}

Twenty nine healthy volunteers (nineteen male, mean age $23.0 \pm 4.9$ years) were recruited from University of Leicester staff and students. Of these, 9 were rejected due to poor quality TCD waveforms (4/9) or disruption of signal during SSMs (5/9). The Velcro thigh-cuff was disrupted in four subjects of the remaining twenty subjects towards the end of the SSMs. Without a reliable marker of the beginning of each squat, it was not possible to produce synchronised averaging of the variables under analysis, thus rendering their data unusable and leaving sixteen data sets for subcomponent analysis.

No significant differences were noted in mean, systolic, or diastolic CBV between the two hemispheres. Mean values across the two recordings are detailed in Table 1. With the exception of diastolic BP, all other haemodynamic parameters showed significant differences between the standing and squatting recordings.

\section{Subcomponent analysis}

In the population coherent averages, $t=0$ s marked the beginning of the squat manoeuvre, whilst $t=10$ s represented the return to the standing position. 
Fig. 1 illustrates the large changes induced by SSM in both peripheral and cerebrovascular parameters. Population coherence averages of peripheral parameters (Fig. 2) show substantial changes in MAP, $\mathrm{HR}$, pulse pressure and end-tidal $\mathrm{CO}_{2}$ following the squat phase, and return to the original values with standing. HR demonstrated an asymmetrical response. Following a rapid drop in response to the rise in MAP, HR started to return to its original value five seconds into the squat position, but with a much slower rate of rise than observed for the sharp drop at the beginning of the squat. Pulse pressure decreased immediately following the SSM, but then increased before decreasing again while the subject was in the squat position (Fig. 2B). As the subject stood pulse pressure increased sharply for four seconds before reducing down towards baseline.

Bilateral cerebral parameters did not show interhemispheric differences as demonstrated in Fig. 3. For this reason, subsequent graphical results for sub-component analysis will refer only to the changes in the left hemisphere, since the majority of our subjects (14/16) were right handed as assessed by the Edinburgh Handedness Inventory.

With the beginning of the squat manoeuvre (Fig. $4, \mathrm{t}=0$ ), both $\mathrm{V}_{\mathrm{MAP}}$ and $\Delta \mathrm{V}$ increased rapidly, but whilst $\mathrm{V}_{\mathrm{MAP}}$ remained approximately stable, $\Delta \mathrm{V}$ showed a marked trend to return to its original value, thus providing a visual indication of an autoregulatory response. These changes were reversed when subjects returned to the standing position (Fig. $4, t=10 \mathrm{~s}$ ). Both $\mathrm{V}_{\text {RAP }}$ and $\mathrm{V}_{\mathrm{CrCP}}$ contributed towards reducing $\Delta \mathrm{V}$ following squatting (Fig. 4B \& D). Although both subcomponents had a delay compared to the $\mathrm{V}_{\mathrm{MAP}}$ change, $\mathrm{V}_{\mathrm{CrCP}}$ showed an asymmetrical response when subjects returned to the standing position with a further drop and a much slower return to baseline than $V_{\mathrm{RAP}}$. Table 2 presents mean $\pm \mathrm{SD}$ values for the subcomponents every five seconds along the averaged $20 \mathrm{~s}$ squat-stand cycle for both hemispheres.

\section{Discussion}

SSMs have been employed in multiple studies of dynamic CA, in both healthy subjects and patients with clinical conditions $(9,36,37)$. To our knowledge, this is the first study to provide 
a time-domain analysis of the manoeuvre, describing the different contributions to the CBV response and their complex interaction with peripheral and cerebral parameters. We have shown that the temporal pattern of the CBV response confirms the presence of active dCA in healthy subjects. This substantiates the more common analytic approach of TFA which, despite providing estimates of gain, phase, and significantly enhanced coherence, could represent spurious values resulting from multiple peripheral and cerebrovascular co-factors varying in synchrony with the frequency of SSM. Moreover, we also demonstrated that the CBV response to squatting is likely to include a significant metabolic component (as measured by $\mathrm{V}_{\mathrm{CrCP}}$ ), in addition to the myogenic activation (as measured by $\mathrm{V}_{\mathrm{RAP}}$ ) in response to the sudden rise in BP when subjects squat (29). This interpretation of the CrCP and RAP changes during SSMs will be discussed below.

The physiology of the SSM has previously been described; as the subject squats, contraction of the leg muscles depletes venous pooling, causing an increase in the effective circulating volume (34). This increase in venous return increases end-diastolic volume and consequently stroke volume, thus increasing BP and CBF (21). The muscle mechanoreflex also contributes to the resultant increase in MAP and CBF (48). Meanwhile, the baroreflex responds to the increased BP by decreasing HR (48). Furthermore, the constriction of vessels by the musculature induces a degree of peripheral ischaemia, predisposing to vasodilation once the subject stands $(32,41)$. Upon subsequently standing, BP falls. Total peripheral resistance is low because of the removal of the muscle pump, the release of vasodilatory metabolites during the squat and the activation of the cardiopulmonary baroreflex in response to a sudden increase in the volume of blood reaching the heart $(40,45,46)$, all of which promote vasodilation. As a result, blood returns to venous pooling. Simultaneously, HR increases due to decreased vagal tone $(6,16,18,32)$. A second, more gradual, increase in HR occurs between five and twelve seconds (46). BP rebounds seven seconds after standing due to sympathetically mediated vasoconstriction, in response to the fall in blood pressure and the subsequent unloading of the baroreceptors $(5,6)$. 


\section{Subcomponent analysis}

Subcomponent analysis allows for the identification of the relative contribution of the different influences on CBV throughout SSMs. Previously, subcomponent analysis has been used to assess changes in CBV in response to other stimuli including mental activation (30) and breath holding (44). Previous work has led to a hypothetical association between changes in RAP as representing the myogenic response, whilst $\mathrm{CrCP}$ would express metabolically driven changes in $\operatorname{CBF}(22,25,29,30,33,44)$. Confirmation of the validity and generalizability of these associations will require more fundamental experimental work. For this reason, caution must be exercised when interpreting our results and our simplistic use of these presumed associations throughout this paper.

Our results demonstrate that $\mathrm{V}_{\mathrm{MAP}}$ increases significantly as the subject squats. This is to be expected, as the change in CBV associated with the SSM is thought to be caused by the large BP oscillations elicited (10). RAP increases throughout the squat as the cerebrovasculature attempts to protect the brain from the increased flow by increasing resistance, and therefore $\mathrm{V}_{\mathrm{RAP}}$ decreases. Similarly, $\mathrm{V}_{\mathrm{CrCP}}$ decreases when the squat manoeuvre is performed, following the rise in $\mathrm{CrCP}$ in real terms during this time. This suggests that the strongest influence of the change in CBV during the SSM is MAP, which overwhelms the metabolic (as measured by $\mathrm{CrCP}$ ) and myogenic (as measured by RAP) autoregulatory processes to be the driving force behind the increased CBV (29). If RAP represents the myogenic response, and if the response of $\mathrm{CBV}$ to the SSM is purely myogenic, RAP should explain the drop in $\triangle \mathrm{V}$ starting three seconds into the squat phase (Fig. 4). As shown in Fig. 5 though, the contribution of $V_{\text {RAP }}$ is not enough to explain the reduction in $\Delta V$ and according to Eq. [8], the difference should be ascribed to the contribution of $\mathrm{V}_{\mathrm{CrCP}}$.

Previous studies employing healthy controls have found the magnitude of $\mathrm{V}_{\text {MAP }}$ to be significantly reduced compared to the current study (30). These studies employed cognitive paradigms, motor paradigms (33) and breath holding (44) to alter CBV. In these studies, $V_{\text {RAP }}$ and $\mathrm{V}_{\mathrm{CrCP}}$ are of a similar magnitude to $\mathrm{V}_{\mathrm{MAP}}$, if not greater. However, in the current study the 
magnitude of $V_{\text {MAP }}$ was much greater than in the previous studies, and much greater than the magnitude of $\mathrm{V}_{\mathrm{RAP}}$ and $\mathrm{V}_{\mathrm{CrCP}}$. Whilst this is not surprising in the context of repeated SSMs when the underlying physiology is considered, it is nonetheless a novel finding in the field of subcomponent analysis.

Fig. 4 demonstrates the response of CBV and its subcomponents to the SSM. By comparing Figs. $4 A$ and $4 C$, we see that CBV returns towards baseline faster than MAP. This may simply illustrate dynamic $\mathrm{CA}$, although it is possible that there are external influences on the response of $\mathrm{CBV}$. End-tidal $\mathrm{CO}_{2}$, which climbs during the performance of the squat (Fig. 2D) is known to reduce $\mathrm{CrCP}$, thus increasing $\mathrm{V}_{\mathrm{CrCP}}$ (25). This is just one example of physiological parameters interfering with the subcomponent response; it is possible that other mechanisms, such as activation of the sympathetic nervous system, cause vasoconstriction to decrease $\mathrm{V}_{\mathrm{CrCP}}$ during the performance of the squat. Furthermore, the asymmetry of $\mathrm{V}_{\mathrm{CrCP}}$ (Fig. 4D) when the subject stands suggests the existence of other external influences on the CBV response. Without these external influences, which may include the muscle mechanoreflex and the activation of the sympathetic nervous system amongst others, it is possible that we may see CBV return towards baseline faster or slower than seen currently. Further work is required to verify this, which may involve autonomic blockade to eradicate the influence of the autonomic nervous system on the CBV response. Previous studies employing autonomic blockade have shown that this impairs the autoregulatory response $(49,51)$. We might therefore expect a reduction in the magnitude of $\mathrm{V}_{\mathrm{CrCP}}$ and $\mathrm{V}_{\mathrm{RAP}}$ and an increase in the magnitude of $\Delta \mathrm{V}$ in such subjects. To our knowledge, the subcomponent response to autonomic blockade has yet to be ascertained; this provides an exciting opportunity for future work.

One input into the complex CrCP response may be a partial Valsalva manoeuvre, which was noted in some participants upon visual inspection of the data. Dawson et al. found CrCP decreased sharply (hence $\mathrm{V}_{\mathrm{CrCP}}$ increased) during phase IV of the Valsalva manoeuvre. This occurs after the strain has been released (11), which may parallel the release of strain after a prolonged squat. At this point, $\mathrm{V}_{\mathrm{CrCP}}$ would be expected to increase in response, but as shown 
in Fig. 4D, $\mathrm{V}_{\mathrm{CrCP}}$ transiently decreases as the subject stands. This transient 'dip' was observed in 14 of the 16 subjects. The reason for this is unclear, but it might have been caused by the complex interaction of multiple co-factors, including the transient increase in $\mathrm{EtCO}_{2}$ (Fig. 2D) and cerebral vasoconstriction due to sympathetic activity.

\section{Oscillations in BP and CBV}

Previous studies employing the SSM have focussed more on TFA and the signal power of the $\mathrm{BP}-\mathrm{CBV}$ relationship, rather than the relevance and interpretation of the physiological response $(9,10,35,37,38)$. Only one study has reported both the change in MAP and CBV during the performance of periodic SSMs (7). Brassard et al reported a change in CBV during the SSM $(0.05 \mathrm{~Hz})$ as $14 \pm 9 \mathrm{~cm} \cdot \mathrm{s}^{-1}$ during squatting, and $-17 \pm 9 \mathrm{~cm} \cdot \mathrm{s}^{-1}$ upon standing. In our study, the mean changes in CBV were $+27.9 \mathrm{~cm} . \mathrm{s}^{-1}$ and $-20.3 \mathrm{~cm} \cdot \mathrm{s}^{-1}$, respectively. The change noted during hypotension is comparable, but in our subjects CBV increased to a much greater degree during the performance of the squat, suggesting that autoregulatory mechanisms during hypotension are more effective than during hypertension. This directly contradicts the findings of Brassard and the findings of other studies assessing the phenomenon of 'hysteresis', which have all found dCA to be more effective in hypertension than hypotension $(1,7,43)$.

Brassard found MAP to fluctuate by $21 \pm 12 \mathrm{mmHg}$ and $-20 \pm 11 \mathrm{mmHg}$ during repeated maneuvers at $0.05 \mathrm{~Hz}(7)$ while Querido reported a maximum change of $+15 \mathrm{mmHg}$ and $13 \mathrm{mmHg}$ during the squat and stand, respectively, but did not report an average oscillation (31). In our study, MAP increased by $35.0 \mathrm{mmHg}$ during the performance of the squat, and decreased by $36.4 \mathrm{mmHg}$ when the subject stood; we also found a relatively symmetrical response to the SSM, but of larger magnitude.

The discrepancy in the direction of hysteresis is most likely due to the reference values used to calculate the magnitude of the oscillations. Brassard used mean values from a separate resting recording of subjects sitting as the reference from which oscillation magnitudes were 
calculated (7). However, we believe that using a separate resting period as the reference point is misleading. Prior to the subject standing $(t=10)$, BP is greatly elevated compared to mean BP at rest. Therefore, when the subject stands, the true size of the oscillation induced is much greater than that calculated when using resting BP as a reference point. This leads us to question which reference values are more reliable to represent the physiological changes taking place during SSMs. In the case of SSMs, we believe using values immediately prior to the maneuvers is more valid than using a separate control recording as they represent the challenge to the cerebrovasculature at that very moment.

\section{Responses of cardiovascular parameters}

The temporal response of MAP to the SSM closely mimics that described by the literature. Classically, MAP is described as decreasing sharply upon standing, and then increasing seven seconds later as the sympathetic nervous system initiates vasoconstriction $(32,46)$. This pattern correlated very closely with the findings of our study.

The observed overall decrease in HR following the performance of the squat is most likely mediated through the baroreflex (24). Shortly following the performance of the SSM, HR increases slightly despite MAP increasing simultaneously, a finding also noted in the literature (24). This may be due to an 'exercise effect', whereby contraction of the leg muscles creates abrupt vagal inhibition, leading to an increase in HR (5). In the literature this exercise reflex is considered when the subject stands up from a single prolonged squat, but it is known that the muscular effort of changing posture elicits the exercise reflex $(5,18)$, and therefore this mechanism is applicable to our repeated maneuvers. An anticipatory mechanism may also be involved; anticipation of exercise, in this case the performance of a prolonged squat, is known to increase both HR and MAP (39). After this initial increase in HR, the initiation of the baroreflex in response to a substantial increase in MAP overrides the exercise reflex, leading to the overall reduction in $\mathrm{HR}$ that we see during the initial portion of the squat. 
Before the squat portion of the manoeuvre is complete, HR begins to increase again (Fig. 2). This occurs prior to MAP decreasing towards baseline, which suggests that the deactivation of baroreflex is not responsible for this initial increase. The reason for this is unclear; it is possible that the exercise effect begins to override the baroreflex as the subject starts to struggle with the exertion of the SSM. One study reported a continuously low HR following the performance of a sustained squat (20), another reported a recovery of HR after $60 \mathrm{~s}$ of squatting (17), but no literature could be found that described an increase in HR prior to a reduction in BP during the SSM.

However, it is known that BP decreases and HR increases prior to the completion of the Valsalva manoeuvre, due to increased intrathoracic pressure limiting cardiac filling, and therefore compromising cardiac output (42). A similar pattern was seen during the latter part of the squat, which may be due to a partial Valsalva manoeuvre when participants exerted themselves, as described above.

The fluctuating response of pulse pressure during the performance of the squat may be due to concurrent changes in HR and MAP (Fig. 2). The increase in pulse pressure from $t=2-5 \mathrm{~s}$ occurs as HR falls simultaneously. This fall HR allows for increased cardiac filling, and consequently increased stroke volume, to generate a greater pulse pressure. As the subject stands, the initial increase in pulse pressure is most likely caused by decreased afterload,(32) as demonstrated by the simultaneous fall in MAP (Fig. 2). Better understanding of the peripheral changes contributing to the cerebrovascular response to SSM might be gained in future studies by estimating the continuous temporal changes in peripheral resistance or conductance, although this would represent a considerable technical challenge using stateof-the-art techniques for accurate continuous measurement of stroke volume.

The asymmetrical response of end-tidal $\mathrm{CO}_{2}$ can perhaps be explained by considering the underlying physiology. During the SSM, the contracting muscles of the leg create a closed system, restricting blood flow to the muscle fibres and thus creating a relative anaerobic environment that strongly favours the production of lactic acid (19). This lactic acid is then 
converted into $\mathrm{CO}_{2}$ and exhaled through the respiratory system. As a result, the rate of $\mathrm{CO}_{2}$ production, and therefore exhalation, during the active portion of the SSM should be greater than during the passive portion, as was found in our study. Another possible explanation is a degree of breath-holding by participants when performing the squat, as noted above. Breathholding will lead to the accumulation of $\mathrm{CO}_{2}$, which is released once the subject exhales. The degree to which this contributes to the asymmetry of the $\mathrm{CO}_{2}$ curve is uncertain, but it is likely to play a role.

\section{Limitations}

We used TCD to measure CBV in the MCAs. Using CBV as a surrogate measure for CBF relies on the assumption that the diameter of the MCA remains relatively constant. If the diameter of the MCA were to change, it would be possible for a change in flow to be overestimated in the case of vasoconstriction or underestimated in the case of vasodilation. Traditional studies considering the influence of $\mathrm{pCO}_{2}$ on the diameter of the MCA have generally shown there to be no significant effect when arterial gases are in normal ranges (47). In this present study, mean end-tidal $\mathrm{CO}_{2}$ values for the four recordings were within 3.3 $\mathrm{mmHg}$ of each other.

In our female volunteers, we have not controlled for the stage of their menstrual cycle and this could potentially be a confounder in the cerebrovascular response to SSM.

\section{Conclusions}

In summary, the time-domain analysis of the SSM we performed has confirmed that the CBV response to the manoeuvre reflects activation of dynamic CA following substantial changes in $\mathrm{BP}$ as the subject squats and then stands. Both myogenic and metabolic components of the dynamic CA response contribute significantly to the CBV changes observed, but more work is needed to clarify the potential contribution of other co-factors, such as breath-to-breath changes in $\mathrm{pCO}_{2}, \mathrm{HR}$, stroke volume and the neurogenic component of dynamic CA. 
Disclosure/conflict of interest

The authors declare no conflict of interest. 


\section{References}

1. Aaslid R, Blaha M, Sviri G, Douville CM and Newell DW. Asymmetric dynamic cerebral autoregulatory response to cyclic stimuli. Stroke. 38: 5: 1465-1469, 2007.

2. Aaslid R, Lindegaard KF, Sorteberg W and Nornes H. Cerebral autoregulation dynamics in humans. Stroke. 20: 1: 45-52, 1989.

3. Birch A, Neil-Dwyer G and Murrills A. The repeatability of cerebral autoregulation assessment using sinusoidal lower body negative pressure. Physiol Meas. 23: 1: 73, 2001.

4. Birch AA, Dirnhuber MJ, Hartley-Davies R, lannotti F and Neil-Dwyer G. Assessment of autoregulation by means of periodic changes in blood pressure. Stroke. 26: 5: 834-837, 1995.

5. Borst C, van Brederode JF, Wieling W, van Montfrans GA and Dunning AJ. Mechanisms of initial blood pressure response to postural change. Clin Sci. 67: 3: 321-327, 1984.

6. Borst C, Wieling W, van Brederode JF, Hond A, de Rijk LG and Dunning AJ. Mechanisms of initial heart rate response to postural change. Am J Physiol. 243: 5: H67681, 1982.

\section{Brassard P, Ferland-Dutil H, Smirl JD, Paquette M, Le Blanc O, Malenfant S and} Ainslie PN. Evidence for hysteresis in the cerebral pressure-flow relationship in healthy men. Am J Physiol Heart Circ Physiol. 312: 4: H701-H704, 2017.

8. Burton AC. On the physical equilibrium of small blood vessels. Am J Physiol. 164: 2: 319329, 1951. 


\section{Claassen JA, Diaz-Arrastia R, Martin-Cook K, Levine BD and Zhang R. Altered}

cerebral hemodynamics in early Alzheimer disease: a pilot study using transcranial Doppler. J Alzheimer's Dis. 17: 3: 621-629, 2009.

10. Claassen JA, Levine BD and Zhang R. Dynamic cerebral autoregulation during repeated squat-stand maneuvers. J Appl Physiol. 106: 1: 153-160, 2009.

11. Dawson SL, Panerai RB and Potter JF. Critical closing pressure explains cerebral hemodynamics during the Valsalva maneuver. J Appl Physiol. 86: 2: 675-680, 1999.

12. Dewey RC, Pieper HP and Hunt WE. Experimental cerebral hemodynamics: vasomotor tone, critical closing pressure, and vascular bed resistance. J Neurosurg. 41: 5: 597-606, 1974.

13. Diehl RR, Linden D, Lucke D and Berlit P. Phase relationship between cerebral blood flow velocity and blood pressure. A clinical test of autoregulation. Stroke. 26: 10: 1801-1804, 1995.

14. Downey JM and Kirk ES. Inhibition of coronary blood flow by a vascular waterfall mechanism. Circ Res. 36: 6: 753-760, 1975.

15. Evans DH, Levene MI, Shortland DB and Archer LNJ. Resistance index, blood flow velocity, and resistance-area product in the cerebral arteries of very low birth weight infants during the first week of life. Ultrasound Med Biol. 14: 103-110, 1988.

16. Goodwin G, McCloskey D and Mitchell J. Cardiovascular and respiratory responses to changes in central command during isometric exercise at constant muscle tension. $J$ Physiol. 226: 1: 173-190, 1972. 
17. Hanson P, Slane PR, Rueckert PA and Clark SV. Squatting revisited: comparison of haemodynamic responses in normal individuals and heart transplantation recipients. $\mathrm{Br}$ Heart J. 74: 2: 154-158, 1995.

18. Hollander AP and Bouman LN. Cardiac acceleration in man elicited by a muscle-heart reflex. J Appl Physiol. 38: 2: 272-278, 1975.

19. Katz A and Sahlin K. Regulation of lactic acid production during exercise. J Appl Physiol. 65: 2: 509-518, 1988.

20. Lance VQ and Spodick DH. Physiological responses to prompt and sustained squatting. Measurement by systolic time intervals. Br Heart J. 39: 5: 559-562, 1977.

21. Lewis BS, Lewis $\mathbf{N}$ and Gotsman MS. Effect of standing and squatting on echocardiographic left ventricular function. Eur J Cardiol. 11: 6: 405-412, 1980.

22. Maggio P, Salinet AS, Panerai RB and Robinson TG. Does hypercapnia-induced impairment of cerebral autoregulation affect neurovascular coupling? A functional TCD study. J Appl Physiol. 115: 4: 491-497, 2013.

23. Micieli G, Tassorelli C, Bosone D, Cavallini A, Viotti E and Nappi G. Intracerebral vascular changes induced by cold pressor test: a model of sympathetic activation. Neurol Res. 16: 3: 163-167, 1994.

24. O'Donnell TV and Mcllroy MB. The circulatory effects of squatting. Am Heart J. 64: 347-356, 1962.

25. Panerai R. The critical closing pressure of the cerebral circulation. Med Eng Phys. 25: 8: 621-632, 2003. 
26. Panerai R, Rennie J, Kelsall A and Evans D. Frequency-domain analysis of cerebral autoregulation from spontaneous fluctuations in arterial blood pressure. Med Biol Eng Comput. 36: 3: 315-322, 1998.

27. Panerai RB. Transcranial Doppler for evaluation of cerebral autoregulation. Clin Auton Res. 19: 4: 197-211, 2009.

28. Panerai RB, Kelsall AWR, Rennie JM and Evans D. Analysis of cerebral blood flow autoregulation in neonates. IEEE Trans Biomed Eng. 43: 8: 779-788, 1996.

29. Panerai RB, Eyre M and Potter JF. Multivariate modeling of cognitive-motor stimulation on neurovascular coupling: transcranial Doppler used to characterize myogenic and metabolic influences. Am J Physiol Regul Integr Comp Physiol. 303: 4: R395-407, 2012.

30. Panerai RB, Moody M, Eames PJ and Potter JF. Cerebral blood flow velocity during mental activation: interpretation with different models of the passive pressure-velocity relationship. J Appl Physiol. 99: 6: 2352-2362, 2005.

31. Querido JS, Ainslie PN, Foster GE, Henderson WR, Halliwill JR, Ayas NT and Sheel AW. Dynamic cerebral autoregulation during and following acute hypoxia: role of carbon dioxide. J Appl Physiol. 114: 9: 1183-1190, 2013.

32. Rossberg F and Peňaz J. Initial cardiovascular response on change of posture from squatting to standing. Eur J Appl Physiol Occup Physiol. 57: 1: 93-97, 1988.

33. Salinet AS, Robinson TG and Panerai RB. Cerebral blood flow response to neural activation after acute ischemic stroke: a failure of myogenic regulation? J Neurol. 260: 10: 2588-2595, 2013.

34. Sharpey-Schafer EP. Effects of squatting on the normal and failing circulation. Br Med J. 1: 4975: 1072-1074, 1956. 
35. Smirl JD, Lucas SJ, Lewis NC, Smith KJ, Bakker A, Basnyat AS and Ainslie PN. Cerebral pressure-flow relationship in lowlanders and natives at high altitude. $J$ Cereb Blood Flow Metab. 34: 2: 248-257, 2014.

36. Smirl JD, Haykowsky MJ, Nelson MD, Tzeng YC, Marsden KR, Jones H and Ainslie PN. Relationship between cerebral blood flow and blood pressure in long-term heart transplant recipients. Hypertension. 64: 6: 1314-1320, 2014.

\section{Smirl JD, Hoffman K, Tzeng YC, Hansen A and Ainslie PN. Methodological} comparison of active- and passive-driven oscillations in blood pressure; implications for the assessment of cerebral pressure-flow relationships. J Appl Physiol. 119: 5: 487-501, 2015.

38. Smirl JD, Tzeng YC, Monteleone BJ and Ainslie PN. Influence of cerebrovascular resistance on the dynamic relationship between blood pressure and cerebral blood flow in humans. J App/ Physiol. 116: 12: 1614-1622, 2014.

39. Smith EE, Guyton AC, Manning RD and White RJ. Integrated mechanisms of cardiovascular response and control during exercise in the normal human. Prog Cardiovasc Dis. 18: 6: 421-443, 1976.

40. Sprangers RL, Wesseling KH, Imholz AL, Imholz BP and Wieling W. Initial blood pressure fall on stand up and exercise explained by changes in total peripheral resistance. $J$ Appl Physiol .70: 2: 523-530, 1991.

41. Tanaka H, Sjöberg B and Thulesius O. Cardiac output and blood pressure during active and passive standing. Clin Physiol. 16: 2: 157-170, 1996.

42. Tiecks FP, Lam AM, Matta BF, Strebel S, Douville C and Newell DW. Effects of the valsalva maneuver on cerebral circulation in healthy adults. A transcranial Doppler Study. Stroke. 26: 8: 1386-1392, 1995. 


\section{Tzeng YC, Willie CK, Atkinson G, Lucas SJ, Wong A and Ainslie PN.}

Cerebrovascular regulation during transient hypotension and hypertension in humans. Hypertension. 56: 2: 268-273, 2010.

44. van Veen TR, Panerai RB, Haeri S, Zeeman GG and Belfort MA. Effect of breath holding on cerebrovascular hemodynamics in normal pregnancy and preeclampsia. J Appl Physiol. 118: 7: 858-862, 2015.

45. Wieling W, Harms M, Ten Harkel A, van Lieshout JJ and Sprangers R. Circulatory response evoked by a $3 \mathrm{~s}$ bout of dynamic leg exercise in humans. J Physiol. 494: 2: 601 $611,1996$.

46. Wieling W, Krediet CT, van Dijk N, Linzer M and Tschakovsky ME. Initial orthostatic hypotension: review of a forgotten condition. Clin Sci. 112: 3: 157-165, 2007.

47. Willie C, Colino F, Bailey D, Tzeng Y, Binsted G, Jones L, Haykowsky M, Bellapart J, Ogoh S and Smith K. Utility of transcranial Doppler ultrasound for the integrative assessment of cerebrovascular function. J Neurosci Methods. 196: 2: 221-237, 2011.

48. Zhang R, Claassen JA, Shibata S, Kilic S, Martin-Cook K, Diaz-Arrastia R and Levine BD. Arterial-cardiac baroreflex function: insights from repeated squat-stand maneuvers. Am J Physiol Regul Integr Comp Physiol. 297: 1: R116-23, 2009.

49. Zhang R, Crandall CG and Levine BD. Cerebral hemodynamics during the Valsalva maneuver: insights from ganglionic blockade. Stroke. 35: 4: 843-847, 2004.

50. Zhang R, Zuckerman JH, Giller CA and Levine BD. Transfer function analysis of dynamic cerebral autoregulation in humans. Am J Physiol. 274: 1 Pt 2: H233-41, 1998. 
51. Zhang R, Zuckerman JH, Iwasaki K, Wilson TE, Crandall CG and Levine BD.

Autonomic neural control of dynamic cerebral autoregulation in humans. Circulation. 106: 14: 1814-1820, 2002. 
Figure legends

Figure 1: Representative recordings for a 22-year-old male demonstrating changes in CBV $(A)$, mean arterial pressure $(B)$, heart rate $(C)$, end-tidal $\mathrm{CO}_{2}(D)$, resistance area product $(E)$ and critical closing pressure $(\mathrm{F})$ during $15 \mathrm{SSMs}$ at $0.05 \mathrm{~Hz}$.

Figure 2: Response of peripheral parameters to repeated squatting $(t=0 \mathrm{~s})$, followed by standing ( $\mathrm{t}=10 \mathrm{~s}$ ); A) MAP, B) Pulse pressure, C) Heart rate and D) End-tidal $\mathrm{CO}_{2}$, , The squat angle relative to horizontal (right axis) is represented as dashed line. Error bars are largest \pm 1 SE at the point of occurrence.

Figure 3: Population mean change in cerebral blood flow velocity (CBV, $A)$, resistance area product (RAP, B) and critical closing pressure $(\mathrm{CrCP}, \mathrm{C})$ in the left (dashed line) and right (continuous line) brain hemispheres during the performance of the SSM. Squatting started at $\mathrm{t}=0 \mathrm{~s}$, followed by standing at $\mathrm{t}=10 \mathrm{~s}$.

Figure 4: Response of the CBV subcomponents in the left hemisphere to the SSM. A) $\Delta \mathrm{V}$, Cerebral blood flow velocity change from beginning of squat $(\mathrm{t}=0 \mathrm{~s})$, followed by standing ( $t=10 \mathrm{~s})$; B) $\mathrm{V}_{\mathrm{RAP}}, \%$ contribution of resistance area product to $\triangle \mathrm{CBV}$; C) $\mathrm{V}_{\mathrm{MAP}}, \%$ contribution of mean arterial pressure to $\triangle \mathrm{CBV}$; D) $\mathrm{V}_{\mathrm{CrCP}}, \%$ contribution of critical closing pressure to $\triangle \mathrm{CBV}$. Error bars correspond to the largest $\pm 1 \mathrm{SE}$ at the point of occurrence.

Figure 5: Population mean change in $\Delta \mathrm{V}$ (left axis, solid line) to squatting ( $\mathrm{t}=0)$ followed by standing ( $\mathrm{t}=10 \mathrm{~s}$ ), compared with the average contributions of $\mathrm{V}_{\text {MAP }}+\mathrm{V}_{\mathrm{RAP}}$ (right axis, dashed line). See text for interpretation and discussion. 
Tables

Table 1: Main physiological parameters during standing and squat-stand maneuvers.

\begin{tabular}{lccc}
\hline Protocol & Standing & SSMs & P-value \\
\hline Right MCA CBV $\left(\mathbf{c m} . \mathbf{s}^{-1}\right)$ & $52.6 \pm 12.4$ & $58.4 \pm 12.3$ & $<0.01$ \\
\hline Left MCA CBV $\left(\mathbf{c m} . \mathbf{s}^{-1}\right)$ & $51.7 \pm 12.1$ & $55.8 \pm 11.2$ & 0.01 \\
\hline Mean arterial pressure & $91.2 \pm 9.9$ & $97.1 \pm 11.7$ & \\
(mmHg) & & & 0.02 \\
\hline Systolic blood pressure & $121.0 \pm 12.2$ & $131.6 \pm 16.8$ & \\
(mmHg) & & & 0.16 \\
\hline Diastolic blood pressure & $79.8 \pm 10.7$ & $83.8 \pm 9.3$ & $<0.01$ \\
(mmHg) & & & $<0.01$ \\
\hline Heart rate $(\mathbf{b p m})$ & $88.3 \pm 12.0$ & $98.1 \pm 11.5$ & \\
\hline End-tidal $\mathbf{C O}_{2}$ (mmHg) & $36.0 \pm 3.0$ & $39.3 \pm 3.4$ & \\
\hline
\end{tabular}

Values are mean \pm SD $(n=16)$ for the entire five min recording. MCA, middle cerebral artery; CBV, cerebral blood velocity; SSM, squat-stand maneuver. $P$ value, paired Student's t-test. 
Table 2: CBV change and its components at selected time intervals after activation $(t=0)$

\begin{tabular}{llllllll}
\hline \multicolumn{3}{c}{ Right MCA } & & & Left MCA & \multicolumn{1}{c}{$\begin{array}{l}\text { Blood } \\
\text { pressure }\end{array}$} \\
\hline Time $(\mathbf{s})$ & $\Delta \mathrm{V}$ & $\mathrm{V}_{\text {RAP }}$ & $\mathrm{V}_{\text {CrCP }}$ & $\Delta \mathrm{V}$ & $\mathrm{V}_{\text {RAP }}$ & $\mathrm{V}_{\text {CrCP }}$ & $\mathrm{V}_{\text {MAP }}$ \\
\hline $\mathbf{0}$ & $2.3 \pm 1.9$ & $-0.4 \pm 1.8$ & $0.2 \pm 2.4$ & $2.4 \pm 2.1$ & $-0.2 \pm 2.1$ & $0.0 \pm 2.7$ & $2.5 \pm 2.3$ \\
\hline $\mathbf{5}$ & $35.7 \pm 21.8$ & $-23.6 \pm 10.9$ & $-24.6 \pm 31.9$ & $36.4 \pm 22.1$ & $-20.3 \pm 12.6$ & $-26.9 \pm 28.0$ & $83.6 \pm 21.1$ \\
\hline $\mathbf{1 0}$ & $12.0 \pm 15.7$ & $-27.3 \pm 12.8$ & $-26.7 \pm 25.8$ & $13.3 \pm 14.7$ & $-24.8 \pm 12.7$ & $-27.4 \pm 22.8$ & $66.2 \pm 39.9$ \\
\hline $\mathbf{1 5}$ & $-20.4 \pm 5.1$ & $0.0 \pm 9.5$ & $10.1 \pm 8.7$ & $-20.8 \pm 5.4$ & $0.3 \pm 10.4$ & $-10.6 \pm 10.3$ & $-10.1 \pm 10.2$ \\
\hline $\mathbf{2 0}$ & $1.3 \pm 1.8$ & $2.0 \pm 3.3$ & $1.3 \pm 3.8$ & $1.6 \pm 1.6$ & $2.6 \pm 3.3$ & $0.9 \pm 2.9$ & $-2.3 \pm 5.7$ \\
\hline \multicolumn{2}{l}{ Values are mean \pm SD. CBV, cerebral blood flow velocity. $\Delta \mathrm{V}$, change in CBV (\%) normalised to absolute values at beginning of squatting. }
\end{tabular}

Values are mean \pm SD. CBV, cerebral blood flow velocity. $\triangle \mathrm{V}$, change in CBV (\%) normalised to absolute values at beginning of squatting. $\mathrm{V}_{\mathrm{RAP}}, \mathrm{V}_{\text {CrCP }}, \mathrm{V}_{\text {MAP }}$ : \% relative contributions of resistance-area product (RAP), critical closing pressure (CrCP) and mean arterial blood pressure (MAP), respectively, to the overall $\Delta \mathrm{V}$ change. 